P1-301 MODELING OF CLINICAL MEASURES TO PREDICT FLORBETAPIR F18 PET RESULTS IN MILD AND MODERATE ALZHEIMER'S DEMENTIA

Michael Witte ${ }^{1}$, Paula Trzepacz ${ }^{1}$, Michael Case ${ }^{1}$, Peng Yu ${ }^{1}$, Helen Hochstetler ${ }^{1}$, Sara Kollack Walker ${ }^{1}$, Karen Sundell ${ }^{2}$, David Henley ${ }^{2}$, ${ }^{1}$ Eli Lilly and Company, Indianapolis, Indiana, United States; ${ }^{2}$ Lilly Research Laboratories, Indianapolis, Indiana, United States. Contact e-mail: witte_michael_m@lilly.com

Background: Alzheimer's disease (AD) is challenging to diagnose clinically, with the sensitivity of clinical criteria applied by experts ranging from $71 \%-87 \% .1$ We sought clinical characteristics that could help predict a negative florbetapir F 18 Positron Emission Tomography (FBPPET) scan in clinically diagnosed AD dementia patients Methods: A subset of patients (199/2648; $\geq 55$ years with clinically diagnosed mild or moderate AD dementia using NINCDS/ADRDA criteria) pooled from 2 anti-amyloid drug trials participated in an optional FBP-PET addendum at pre-randomization. A composite cortical standardized uptake value ratio (SUVr) $<1.1$ using whole cerebellum as the reference region was categorized as a negative scan. Using a forward selection multivariate model building process from a logistic regression, we considdered 19 demographic and clinical variables for their association with a positive/negative scan. These included the Alzheimer's Disease Assessment Scale-cognitive subscale 14-item (ADAS-Cog14), Alzheimer's Disease Cooperative Study Activities of Daily Living Inventory (ADCS-ADL), Clinical Dementia Ration (CDR-sum of boxes), Mini Mental-State Exam (MMSE), Neuropsychiatric Inventory-12 item (NPI-12), and Geriatric Depression Scale (GDS). Results: In this clinically diagnosed sample, $35(18 \%)$ patients were FBP-PET negative for amyloid plaque. Previously, using univariate analysis, patients with an FBP-PET positive scan were significantly more impaired on ADAS-Cog14 $(\mathrm{p}<.001)$ and MMSE $(\mathrm{p}<.001)$, but less impaired on GDS $(\mathrm{p}=.010)$; more likely to be categoried as having moderate dementia $(\mathrm{p}=.003)$, and carriers for an ApoE-4 allele $(\mathrm{p}<.001)$. Using multivariate analysis, patients with an FBP-PET negative scan were associated with significantly higher GDS and MMSE scores, although protocol inclusion criteria limited those score ranges (GDS $\leq 6$; MMSE=16-26). Overall, significantly more ApoE-4 allele non-carriers had FBP-PET negative scans, while more ApoE-4 homozygote patients had FBP-PET positive scans. The multivariate model correctly predicted positive and negative scans at 83.9\%. Conclusions: Though diagnosed with AD dementia, $18 \%$ had an FBP-PET negative scan inconsistent with a diagnosis of AD. While our model identified several clinical variables associated with a negative FBPPET scan, these variables did not allow a more reliable prediction of which dementia patients are likely to have Alzheimer's disease. This study highlights the importance of amyloid PET neuroimaging in the dementia differential diagnostic process.

\section{P1-302 THE STABILITY OF RESTING STATE NETWORKS IN AGING: RELEVANCE FOR EARLY MEMORY DYSFUNCTION}

Lies Clerx ${ }^{1}$, Juraj Kukolja ${ }^{2}$, Yasemin Göreci ${ }^{3}$, Anne Hafkemeijer ${ }^{4}$, Oezguer Onur ${ }^{5}$, Pauline Aalten ${ }^{6}$, Frans R. J. Verhey ${ }^{1}$, Gereon R. Fink ${ }^{7}$, Heidi Jacobs ${ }^{1},{ }^{1}$ Maastricht University, Maastricht, Netherlands; ${ }^{2}$ Forschungszentrum Jülich GmbH/Institute of Neuroscience and Medicine-3, Jülich, Germany; ${ }^{3}$ University Hospital of Cologne, Cologne, Germany; ${ }^{4}$ Institute of Psychology, Leiden University, Leiden, Netherlands; ${ }^{5}$ Forschungszentrum Juelich/Institute of Neuroscience and Medicine-3, Jülich, Germany; ${ }^{6}$ MUMC+, Maastricht, Netherlands; ${ }^{7}$ Forschungszentrum
Jülich/Institute of Neuroscience and Medicine-3, Jülich, Germany. Contacte-mail: l.clerx@maastrichtuniversity.nl

Background: The default mode network (DMN) is one of the most investigated resting state networks (RSNs). Reduced functional connectivity (FC) and atrophy in hubs of the DMN is a consistent finding in neurodegenerative diseases such as Alzheimer's disease (AD). Therefore, the DMN has been suggested as a candidate biomarker for AD. In order to define $\mathrm{FC}$ changes in the $\mathrm{DMN}$ as a biomarker for $\mathrm{AD}$, its stability in normal aging needs to be assessed. The present study investigated the test-retest-reproducibility (i.e. stability) of RSNs within and between healthy old (HO) and healthy young (HY) subjects over 3 different time-points. Additionally it was investigated whether individual changes in stability are associated with cognitive performance. Methods: Twenty HY (age between 23-29 years) and twenty HO (age between 54-66 years) subjects underwent 3.0 Tesla MRI scanning. Three scan sessions were administered with on average two weeks in between each session. Differences between groups and over time in FC in eight of the most reliable RSNs, including the DMN, were investigated by applying dual regression methods (FSL). Results: Within group analyses showed no changes over time in any of the investigated RSNs. Between group comparisons showed decreased FC in the DMN and the auditory system in $\mathrm{HO}$ subjects compared to HY subjects. This pattern was consistent over the three time-points. On an individual-level, significant inverse correlations were found between change in FC of specific DMN areas and neuropsychological test performance: subjects with higher variability in FC over time in the precuneus and the hippocampus showed lower performance on memory tests. Conclusions: $\mathrm{FC}$ in the DMN and the auditory system decreases with aging. Within HY and HO subjects, activity in major RSNs remains stable over time. Instability of FC between both the precuneus and the hippocampus with the rest of the DMN are early indicators of subtle cognitive failure on neuropsychological tests. Stability of FC in certain hubs of the DMN could become a predictor for future memory problems on an individual level. This study shows that investigating the DMN by means of resting state fMRI is valuable for early diagnosis and the development of interventions in at-risk individuals.

\section{P1-303 SCANNER RESOLUTION EFFECTS ON PITTSBURGH COMPOUND B AND FLORBETAPIR QUANTITATIVE MEASUREMENTS}

Gregory Klein ${ }^{1}$, Susan Landau ${ }^{2}$, David Scott ${ }^{3}$, Vahan Sharoyon ${ }^{3}$, Joonmi $\mathrm{Oh}^{4}$, Robert Koeppe ${ }^{5}$, Joyce Suhy ${ }^{4},{ }^{1}$ Synarc Inc., Newark, California, United States; ${ }^{2}$ University of California, Berkeley, Berkeley, California, United States; ${ }^{3}$ Synarc Inc., Newark, California, United States; ${ }^{4}$ Synarc Inc., San Francisco, California, United States, ${ }^{5}$ University of Michigan, Ann Arbor, Michigan, United States. Contact e-mail: gregory. klein@synarc.com

Background: Quantitative amyloid Standard Uptake Value Ratio (SUVr) assessments are used for longitudinal efficacy endpoints in clinical trials, and potentially for cross-sectional evaluation of patient amyloid burden, yet the effect of differing spatial resolution from PET scanners in a multicenter study remains unclear. This investigation quantifies SUVr changes due to spatial resolution difference for Pittsburgh Compound B $(\mathrm{PiB})$ and florbetapir PET data. Methods: Quantitative analysis of ADNI PiB and florbetapir images were completed for a subset of normal, $\mathrm{MCI}$ and $\mathrm{AD}$ subjects scanned with the highest resolution scanners in the ADNI study. Differences in global cortical SUVrs were compared as data were increasingly smoothed. Each scanner's resolution was calibrated from Hoffman phantom acquisition data, and reconstructed images were smoothed to values representing effective resolutions between $5.5 \mathrm{~mm}$ and $9.0 \mathrm{~mm}$ FWHM. Global cortical amyloid retention ratios were calculated using four cortical regions 
defined on co-registered MRI data via Freesurfer, and normalized using both whole cerebellar and cerebellar grey reference regions. Results: A nearly linear shift of SUVr values are seen with increased smoothing, but the direction of effect depends upon the amyloid burden of the subject. Generally, SUVr values increase with smoothing for low-burden subjects, and decrease with smoothing for high-burden subjects. Comparing SUVrs computed at the highest available spatial resolution to those computed at $9 \mathrm{~mm}$ effective resolution, SUVrs changed from -5 to $8 \%$ for florbetapir and $-7 \%$ to $5 \%$ for PIB using whole cerebellar reference regions. Changes due to smoothing using a cerebellar grey reference were similar. Changes due to spatial resolution differences in longitudinal SUVr measurements were fairly small, presumably in part due to the small change in SUVr between time points. Conclusions: White matter partial volume effects appear to increase SUVrs following smoothing in amyloid negative patients, while CSF partial volume effects appear to decrease SUVrs in amyloid positive patients, especially in cases with atrophy. Quantitative SUVr thresholds used for cross-sectional analysis therefore require attention to spatial resolution to compensate for effects of different scanner resolutions. For small longitudinal changes in SUVr, spatial resolution differences appear to have a small effect.

\section{SUVr \% Change at 9mm Smoothing (florbetapir)}

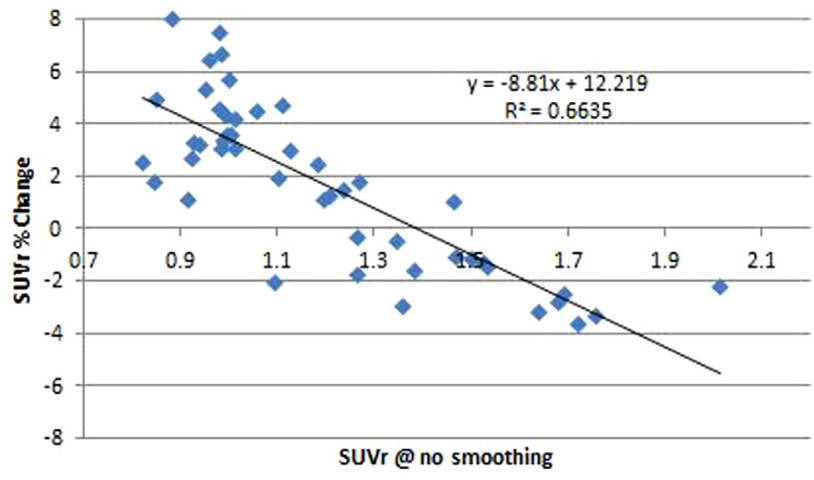

SUVr \% Change at $9 \mathrm{~mm}$ Smoothing

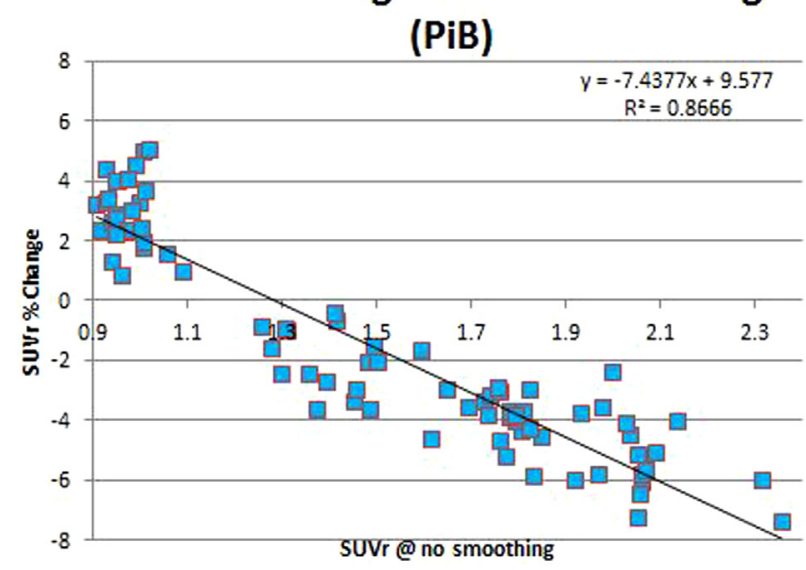

Fig 1. Percent change of composite SUVr for florbetapir (top) and PiB (bottom) ADNI images analyzed at native spatial resolution compared to images smoothed to $9 \mathrm{~mm}$ effective resolution. A trend is seen where increasingly amyloid negative values show increasing SUVr due to smoothing, and conversely, increasingly amyloid positive values show decreasing SUVr due to smoothing.

\section{P1-304 ARTERIAL SPIN LABELING CEREBRAL BLOOD FLOW AND BRAIN VOLUMES IN DEMENTIA-} FREE ELDERLY

Lisa Silbert ${ }^{1}$, David Lahna ${ }^{1}$, William D. Rooney ${ }^{1}$, Hiroko Dodge ${ }^{2}$, Jim Pollaro ${ }^{1}$, Jeffrey Kaye ${ }^{1},{ }^{1}$ Oregon Health \& Science University, Portland, Oregon, United States; ${ }^{2}$ Oregon Health \& Science University/University of Michigan Health System, Portland, Oregon, United States.Contacte-mail: silbertl@ohsu.edu

Background: Decreased brain perfusion, as shown by SPECT imaging, is associated with increased risk of cognitive decline and dementia. Noninvasive measurement of cerebral blood flow (CBF) using arterial spin labeling (ASL) MRI provides an opportunity to examine how CBF may lead to key neurodegenerative changes (e.g., brain volume loss, high WMH burden) associated with increased risk of dementia. In this context, we examined ASL $\mathrm{CBF}$, as a potential functional marker of increased neurodegenerative risk in nondemented elderly. Methods: 49 non-demented volunteers (mean age 85, MMSE 28.5) underwent 3T MRI (Siemens Trio), including structural and Q2TIPS PASL sequences and detailed cognitive and neurological assessment. Automated WMH volumes were obtained from FLAIR images using a customized routine, segmented into deep and periventricular WMHs and $\log$ transformed when necessary to account for skewed distributions. Brain, hippocampal, and WM volumes were obtained using FreeSurfer 5.1. WMHs were subtracted from total WM to obtain normal appearing white matter (NAWM) masks, which were co-registered, along with cortical and subcortical gray matter maps to M0 ASL sequences to obtain regional CBF values $(\mathrm{ml} / 100 \mathrm{~g} / \mathrm{min})$. Differences in total WMH and NAWM CBF were examined using matched pairs t-tests. Linear regressions determined associations between CBF, brain volumes, and subject characteristics. Multivariate linear regression analyses examined relationships between regional $\mathrm{CBF}$ and brain volumes, adjusted for relevant variables. Results: Decreased WM and NAWM CBF, but not cortical or subcortical GM CBF, were associated with increased age, and hypertension (HTN). CBF measures were not associated with MMSE. NAWM CBF was significantly higher than total WM CBF. After adjusting for age, gender, HTN, and intracranial volume, Greater PV, but not deep, WMH was associated with decreased WM and NAWM $\mathrm{CBF}$; decreased total brain volume was associated with lower cortical $\mathrm{CBF}$; and decreased hippocampal volume was associated with higher subcortical CBF. Conclusions: Regional ASL-derived CBF is associated with brain volume change in nondemented elderly at risk for dementia. The inclusion of ASL with structural MRI may enhance the ability to detect those at risk for cognitive decline, and provide a functional biomarker in prevention and treatment trials aimed at improving cerebral perfusion in the elderly.

\section{P1-305 THE PRACTICAL UTILITY OF AMYLOID AND FDG-PET IN AN ACADEMIC DEMENTIA CENTER}

Pascual Sánchez Juan ${ }^{1}$, Jayne Hagen ${ }^{2}$, Pia Ghosh ${ }^{3}$, Benno Gesierich ${ }^{2}$, Maya Henry ${ }^{2}$, Maria-Luisa Gorno-Tempini ${ }^{2}$, Bruce Miller ${ }^{2}$, William Jagust ${ }^{4}$, Gil Rabinovici ${ }^{2},{ }^{1}$ University Hospital Marqués de Valdecilla, Santander, Spain; ${ }^{2}$ UCSF Memory \& Aging Center, San Francisco, California, United States; ${ }^{3}$ UCSF Memory and Aging Center, San Francisco, California, United States; ${ }^{4}$ University of California, Berkeley,Berkeley, California, United States.Contacte-mail: psanchez@ humv.es

Background: We evaluated the impact of amyloid and FDG PET on clinical decision making in a heterogeneous population of cognitively impaired patients. Methods: We selected all patients with FDG and PIB PET and had at least one clinical evaluation before and after PET. We evaluated for change in primary diagnosis and change in treatment between the pre- and post-PET visit. The association of discordant PIB and FDG with changes in clinical management was assessed separately using chi-square and together applying logistic regression. Results: 140 cognitively impaired patients were included in thestudy (mean age $65.0 \pm 8.2,46 . \%$ primary $\mathrm{A} \beta$ diagnosis, $55.0 \%$ of new patients, mean MMSE $22.7 \pm 9.0,41.0 \% \mathrm{CDR}<1$ ). PIB and FDG PET agreed in classifying $83.6 \%$ of patients. Concordance with 